\title{
Der Arzt als Zeuge des Lebens
}

Heinrich Buess zum siebzigsten Geburtstag

Von Heinrich Schipperges

\section{Einführung}

Den Topos vom Arzt als dem «Zeugen der Szenen des Lebens» verdanken wir dem Bonner Kliniker Christian Friedrich Nasse (1778-1851). Das anschauliche Bild läßt sich zurückverfolgen bis auf älteste Zeiten und entspricht einer besonders eindrucksvollen archaischen Leitfigur des ärztlichen Denkens und Handelns. Der Arzt hat den elementaren Szenen des Lebens - der Geburt, dem Sterben und allen kritischen Phasen zwischendurch - immer nahegestanden; er ist nicht nur der getreueste Beobachter der Leiden, sondern auch der beständigste Begleiter der Kranken gewesen. Er ist der Fachmann für Leiden, und er wendet die Not.

Wir beschränken uns bei dieser historischen Skizze - Heinrich Buess zu Ehren und in dankbarer Verbundenheit - auf drei Bereiche: 1. auf einige wenige Bilder und Figuren in der antiken und mittelalterlichen Heilkunde, 2. das Arzt-Patienten-Verhältnis bei Paracelsus und seine exemplarische Zeugenschaft und 3. auf den Arzt als einen Zeugen des Lebens bei Friedrich Nasse. Abschließend wollen wir versuchen, die Möglichkeiten und auch die Grenzen eines solchen Leitbildes für die moderne Medizin aufzuzeigen.

\section{Zeugen des Lebens in Antike und Mittelalter}

Als ein fachkundiger Interpret des «logos» von «bios» begegnet uns der Arzt zum ersten Male in voller Deutlichkeit in der antiken Medizin. Mit der biographischen Szenerie weiß vor allem der hippokratische Arzt immer auch das soziale Fluidum zu verbinden, wobei ein Feld das andere - oft bis in den Wortlaut hinein - zu spiegeln vermag. In den hippokratischen Schriften ist immer wieder von einer Medizin die Rede, die um das humorale Fließgleichgewicht des Organismus kreist, um auszugleichen und zu regulieren. Die Medizin ist gleichsam eine universale Proportionskunde und von daher der Musik so verwandt. Sie erfaßt alle Mißstimmungen und bringt sie über eine 
Konsonierung zur Harmonie, zu einer Isonomie und Eukrasie. Der Arzt ist der Moderator, der Maß nimmt und auch Maßstäbe setzt. Er gleicht die Verluste der Gewichte wieder aus; er kennt die Mitte und weiß um die Grenzen: Er ist der Steuermann, der Kybernetes einer humanen Lebensführung.

Mit seinem Patienten wie mit einem Partner umzugehen, ist nach Platon geradezu das Wesen eines freien Arztes. «Der Arzt teilt seine Eindrücke dem Kranken und dessen Freunden mit, und während er sich über den Kranken unterrichtet, belehrt er ihn gleichzeitig, soweit dies möglich ist. Er verschreibt ihm nichts, ohne ihn zuvor davon überzeugt zu haben. Und so, mit der Hilfe der Unterredung und der Überredung, dem guten Ratschlag auch, beruhigt der Arzt seinen Patienten und bringt ihn dadurch erst in einen Zustand, der es ermöglicht, ihn nach und nach der Gesundheit zuzuführen.» Wer seinen Patienten dagegen nur traktiert und ihm seine Verordnungen zudiktiert, der ist kein freier Arzt, sondern ein Sklaven-Arzt. Ihm liegt nichts an einem Sinn von Leid; er verzichtet auf das Gespräch. Er stellt den Kranken lediglich wieder her: macht ihn leistungsfähig und genußfreudig. Er ist - so würden wir heute sagen - der Kesselflicker des Kapitals!

In seinen «Gesetzen» gibt Platon aber auch zu bedenken: «Wenn einmal ein solcher praktischer Arzt, der nur durch Probieren ohne Wissenschaft die Medizin rein praktisch betreibt, einen nur theoretisch gebildeten Arzt erwischt, der sich mit einem gebildeten Patienten unterhält, dabei aber in seinen Reden geradezu philosophiert, indem er die Erkrankung in ihrem ersten Grunde anfaßt, dann hinaufsteigt zu einer Belehrung über die Natur des Körpers und dergleichen, dann würde der erstere bald in ein lautes Lachen ausbrechen und sagen: «Einfältiger Mensch〉, würde er sagen, «das heißt ja nicht den Kranken kurieren, sondern ihm Lektionen erteilen! Als ob er, der Kranke, ein Doktor werden sollte und nicht vielmehr ein gesunder Mensch!ı »

Auch hier steht uns sehr lebendig die Szenerie des Alltags vor Augen. Der hippokratische Arzt, als ein Diener der Natur, verstand sich darin eher als ein Steuermann (kybernetes). Steuern aber heißt: ein Gleichgewicht halten, das innerhalb eines Spielraumes schwankt, dabei immer wieder an die Grenzen stößt, um zurückzuschwingen auf eine labile Mitte. Steuern meint weniger das robuste Eingreifen als ein behutsames Lenken; im Lenken wird immer nur die Richtung der Fortbewegung bestimmt, aber auch das schwankende Gleichgewicht ausbalanciert. Es ist jene Ordnung der Gesundheit damit gemeint, die mit dem privaten auch den öffentlichen Haushalt in 
Gang hält («oikonomia» in einem «oikos»!). In allem muß der Arzt daher den «kairos» erkennen, um am Fall therapieren zu können; es ist damit eine Therapie angesprochen, die ganz und gar konzentriert ist auf die tagtägliche lebenslange Begleitung des Individuums und damit auch der Gesellschaft.

Eine solche beständige Begleitung der alltäglichsten Erscheinungen des Lebens schien den alten Ärzten besonders schwierig zu sein in einer Kunst, die so vielerlei Aspekte hat wie die Medizin. Die Heilkunst hat gerade nicht die verbindlichen Parameter einer exakten Naturwissenschaft; sie kennt weder Zahl noch Gewicht; ihr einziges Kriterium ist die Reaktion des Patienten. Bei allem Streben nach objektiver Genauigkeit dominiert hier das Subjekt, die Individualität des Kranken, das biographische Szenarium. Im christlichen Mittelalter, so bei Isidor von Sevilla, wird der Arzt zum Moderator humaner Lebensführung. Für Paracelsus ist der Arzt der, «der die Not wendet». Aristoteles hatte dieses universelle Wissen und Wirken auf die Formel gebracht: Der Arzt ist der Logos der Gesundheit. Nur so konnte der Arzt als der Träger eines speziellen Wissens den allgemeinen Lebensstil verantwortlich aufbauen. Damals wurde die Medizin zur führenden Kulturmacht, zu einem Bestandteil der allgemeinen Bildung, so in der griechischen "paideia», so vor allem bei den arabischen Arztphilosophen.

Der konkrete Kontakt mit den großen wie kleinen Krisen des Kranken gilt in der arabischen Medizin geradezu als das Kriterium einer wahren Heilkunst. Die islamische Überlieferung ist voll von Anekdoten über diesen Umgang mit Patienten. Wenn einer einen Kranken besucht und sein Leiden begleitet, dann steigen tausend Engel zu ihm hernieder. Krankenbesuch ist nicht nur ein Zeichen von Frömmigkeit, sondern auch von feiner Bildung (adab). Als fortschrittlich wird in diesem arabischen Mittelalter gerade derjenige Arzt gepriesen, der die Problemlage des Kranken als eines bedürftigen - und damit bevorzugten - Mitmenschen möglichst genau kennt und sich allein damit schon das Vertrauen seines Patienten erwirbt. Der erfahrene Arzt, so lesen wir, bewahrt seinen Partner vor Angst und Schwierigkeiten; er trifft aus seinem Fachwissen heraus Vorsorge für alle seine Lebensbelange.

Aus solchen therapeutischen Prinzipien ergeben sich oft, was wiederum nur an Beispielen angedeutet sei, erstaunliche kasuistische Folgerungen. «Das ist doch nicht die Möglichkeit» - ruft ein christlicher Arzt aus, als er den Harn eines muslimischen Kaufmanns untersucht -: «Das kann doch nicht der Urin eines religiös geordneten Menschen sein!» Er besucht den Kranken, untersucht ihn klinisch durch, um sich dann zu äußern: «Das hätte ich nie für 
möglich gehalten, daß so etwas bei einem gläubigen Menschen vorkäme: Dies ist ja ein Mann, dessen Leber die Sorge verzehrt hat!»

Über die Anlagen und Fähigkeiten eines solchen «Zeugen des Lebens» informiert uns Ahmad b. ${ }^{\mathrm{C}}$ Umar b. ${ }^{\mathrm{c}} \mathrm{Al} \overline{\mathrm{i}}$, ein Hofarzt, Poet und Astrologe im Samarkand des 12. Jahrunderts: «Ein Arzt muß feinfühlig in seiner Veranlagung sein und klug von Natur, hervorragend an Verstandesschärfe. Darunter versteht man eine Schnelligkeit des Geistes, korrekte Urteile zu bilden, um sozusagen einen raschen Übergang vom Unbekannten zum Bekannten zu finden. Kein Arzt aber kann eine solche feinfühlige Veranlagung halten, wenn er den Adel der menschlichen Seele nicht kennt, noch kann er von Natur aus klug sein, wenn er nicht mit der Logik vertraut ist. Und wer nicht rasch ist in der Urteilsbildung, der kann weder zu einem korrekten Verständnis irgendeiner Krankheit noch zu einer wegkundigen Begleitung des Kranken kommen.»

\section{Zum Arzt-Patienten-Verhältnis bei Paracelsus}

Das persönliche Verhältnis des Arztes zu seinem Kranken und damit auch eine exemplarische Zeugenschaft dieser anthropologischen Grundfigur hat im Übergang vom Mittelalter zur Neuzeit Paracelsus (1492-1541) besonders eindrucksvoll herausgearbeitet. Theophrastus von Hohenheim, der sich später Paracelsus nannte, hat in einer einmaligen Weise die anthropologische Voraussetzung eines solchen Verhältnisses betont. Da ist der Mensch als solcher schon zunächst einmal ein «Mängel-Wesen», hilfsbedürftig und hilfesuchend, «zum Umfallen geboren». Der Mensch kann nun einmal nicht «sein eigener Hirt» sein; er bedarf eines Helfers, ist angewiesen auf einen anderen. Der Kranke braucht daher in seiner elementaren Not fundamentale Hilfe; diese leistet ihm mit seinem helfenden Eingriff jener Arzt, der die kleinen wie die großen Szenen des Alltags eindrucksvoll erfährt.

Nun hat es aber - meint Paracelsus - zu allen Zeiten schon zweierlei Sorten von Ärzten gegeben, «solche, die aus Liebe handeln, und solche, die ihren Eigennutz betreiben». Die ersteren nennt Paracelsus «Lammärzte», und dies im Hinblick auf Johannes den Täufer, der wiederum auf Christus hinwies, das "wahre Lamm», auf den «Christus Medicus». «Denn wie ein Lamm und Schaf soll der Arzt sein, der da von Gott ist: Wie ein Wolf aber ist der, der wider Gott seine Heilkunst gebraucht.» Das sind jene «Wolfsärzte», die sich verhalten wie reißende Wölfe: «Sie schneiden aus Lust, rein zur 
Vermehrung ihres eigenen Nutzens, und verachten das Liebesgebot.» Sie arzneien, obwohl sie genau wissen, daß sie nichts können. «Und wie ein Schaf in des Wolfs Rachen, also sind diese Kranken in des Arztes Hand.»

Paracelsus geht in der notwendigen Interaktion zwischen Therapeut und Klient so weit, daß er fordert: Ein Arzt soll seinem Patienten nachbarlich zugetan sein, ja ehelich verbunden sein. Er soll ihn Tag und Nacht vor Augen haben und bedenken, daß er berufen ist, die Not zu wenden. Daher liegt auch - so Paracelsus - an einem Arzte mehr als an allen übrigen Fakultäten. Nach ihm erst kommt der Theologe, der um den Leib wissen soll, ehe er das Heil predigt. Danach erst der Jurist, der diese edle Kreatur nicht wie eine Sau betrachten und wie ein Kalb verurteilen soll, danach erst der Lehrer, der um das göttliche Bild im Menschen wissen muß, das er zu bilden hat. Die Medizin ist deshalb der Exponent aller Fakultäten: Sie ist «der Eckstein der Universität»! Wo auch gäbe es - schließt Paracelsus - noch etwas Wichtigeres auf Erden, als daß man seinem Nächsten Liebe erweist, indem man seine Schmerzen und sein Leiden lindert durch die «Kraft der Arznei»? Und weiter: «Das größte Perlein und der edelste Schatz ist die Heilung, so in der ganzen Heilkunst vermittelt wird, und es ist nichts auf Erden, das größer sei, als die Kranken zu heilen.» Paracelsus bringt dieses Grundverhältnis auf die Formel: «Selig der Kranke, dem Gott zufüget seinen Arzt.»

Aus der Vielfalt eines solchen Umgangs mit Kranksein haben sich dann freilich immer wieder im Laufe der Geschichte einseitige Handlungsmuster in den Vordergrund gespielt, die oft zu ganz extremen Modellen des ärztlichen Denkens und Handelns geführt haben: zu einem mehr individualistisch-humanistisch eingestellten Verhalten (in der Ich-Du-Beziehung zwischen Arzt und Patient) oder aber einem eher sozio-ökonomischen Handlungsmodell (in der Experten-Beziehung zwischen Therapeut und Klient). Im Sinne des ersten, eher personalen Grundmusters aber war der Arzt noch bis in die Mitte des vorigen Jahrhunderts hinein der «Zeuge der großen und kleinen Szenen des Lebens», Zeuge von Geburt und Tod, von allen Hoch- und Tiefzeiten und jeder Lebenskrise. Wo wäre auch sonst in unserer Welt noch ein Beruf zu finden, der so nahe der Zeuge des Lebens wäre und der so konkret einzugreifen wüßte! 


\section{Der «Zeuge des Lebens» bei Friedrich Nasse}

Damit sind wir in unserer knappen historischen Skizze bereits im vergangenen Jahrhundert angekommen, das uns noch so nahe auf den Leib zugeschnitten ist, daß es uns eher als Vorraum erscheint denn als Geschichte. Bis weit in das 19. Jahrhundert hinein wußte der praktische Arzt sich noch ein Bild vom gesunden Menschen und seinen alltäglichen Lebensverhältnissen zu machen, ehe er sich mehr und mehr auf reine Krankheiten oder soziale Abnormitäten konzentrierte. Nicht von ungefähr war der Arzt der «Zeuge der Szenen des Lebens» und damit der Begleiter einer umfassenden Alltagskultur, die in kleinen Gruppen tagtäglich - durch Hausbesuche, als Laienmedizin, in der Korrespondenz, bei Tischgesprächen, im nachbarlichen Austausch - stilisiert und zivilisiert werden konnte.

In diesem Sinne verstanden es die alten Ärzte durchaus, den humanistischen Begriff einer «civilitas» medizinisch zu interpretieren, weshalb man denn auch - so noch während der ganzen Goethezeit - von einer «Lebenskunst» hat sprechen können, von einer «Lebensordnungslehre» und einer «Lebenskultur». Makrobiotik, Eubiotik, Orthobiotik, Kalobiotik - waren nicht nur Titel, Texte und Themen der Kunst, möglichst lange gesund zu leben, sondern auch einer Kunst, dieses Leben zu bereichern, zu vertiefen, zu verschönern und dadurch sinnvoll zu machen.

Das Bild vom «Zeugen des Lebens» geht in einem größeren Zusammenhang erst auf den Bonner Kliniker Christian Friedrich Nasse zurück, und es lohnt sich auch heute noch, diesem System einer Heilkunde einmal systematischer nachzugehen! Den damals dominierenden philosophischen Spekulationen seiner Zeit stellte Nasse ganz bewußt eine empirische Menschenkunde gegenüber, für die er zunächst das rechte Wort nicht fand, die er mit Namen belegte wie Psychophysiologie und Somatopsychologie - dem Sinne nach auch: Soziopsychosomatik - und für die er schließlich seinen Namen nennt: Anthropologie - die «Lehre von dem ganzen Sein und Leben des Menschen».

Christian Friedrich Nasse wurde am 18. April 1778 als Sohn eines Kreisphysikus in Bielefeld geboren, studierte Medizin in Berlin und Halle, wo J. Ch. Reil, J. Fr. Meckel und K. P. J. Sprengel seine Lehrer wurden. Nach langjähriger ärztlicher Praxis wird Nasse 1815 nach Halle berufen und 1819 an die neubegründete Universität in Bonn, wo er mehr als dreißig Jahre gewirkt hat. Während seine Werke - vor allem auch die umfangreichen Zeitschriftenbeiträge - nahezu unbekannt blieben, sind seine Verdienste für den klinischen Unterricht immer wieder hervorgeschoben worden. 
Vor allem im klinischen Unterricht wuchs damals schon das, was man später die «Nasse-Schulung» genannt hat, nämlich: 1. die eigene und lange Beobachtung von einigen wenigen Patienten, die verhindert, daß theoretische Konzeptionen zu einem iatrogenen Artefakt erstarren oder gar in gußeiserner Form hospitalisiert werden, und 2. das umfassende und sorgfältige Eingehen auf alle Lebensumstände eines jeden Kranken, das allein die optimale Atmosphäre für einen vielfältig gestaffelten Indikationsgang und damit auch für den ärztlichen Eingriff garantiert. Und da die «gezwungene Passivität» eines bloß stillen Zuschauers zu Unaufmerksamkeit verlocken müsse, organisiert Nasse ein aktives «bed-side-teaching», eine harte Schule sicherlich, wie Nasse zugibt, aber ein Prüfstein auch über längere Zeit, die man dem werdenden Arzte einfach zumuten müsse, ehe man ihn zu einem «Rezeptschreiber» macht.

Aufgabe der Ausbildung ist es demnach, den ganzen Arzt zu bilden und nicht nur einen Basisarzt, jenen multipotenten Embryodoktor unserer Approbationsordnung, dem verantwortlich einzugreifen gar nicht mehr zugetraut wird. Was aber wäre wohl ein solcher Arzt? Nasse nennt ihn den psycho-somatisch gebildeten Vollarzt, oder mit seinem Spezialbegriff: den Anthropologen. Ärztliche Praxis nämlich - eine Theorie der Therapeutik wäre auf Dauer gar nicht durchzuführen, stünde dahinter nicht auch eine Theorie vom Menschen selber. Während aber die Erforschung der Natur gewaltige Fortschritte gemacht habe, schreite die Erforschung unserer geistigen Natur nur langsam fort. «Gerade an einer so reich ausgestatteten Natur, wie der des Menschen, mußte am meisten zu teilen sein, und so zerfiel sie denn an Psychologen, Zoologen, Physiologen, und Anatomen; ein jeglicher nahm sein Teil.» Warum aber - fragt Nasse weiter - muß gerade der, «welcher über sich selbst Belehrung sucht, jedesmal bei verschiedenen Fakultäten anfragen?» Warum hört man denn nirgendwo ganz «die Lehre von dem ganzen Sein und Leben des Menschen?» Wo in aller Welt sollte man auch «die ganze Menschennatur wissenschaftlich erkennen», wenn nicht in der Medizin!

An diesem entscheidenden Punkt scheint für den Kliniker Nasse des Jahres 1823 die Medizin nun endlich angekommen: «Das Leben der Anthropologie ist erwacht, aber es hat sich noch keinen Organismus gebildet.» Gegenstand dieser Medizinischen Anthropologie wäre der Mensch «in jeder Gestalt seines Daseins», das Kind wie der Greis, Mann wie Frau, der Kranke und der Gesunde, jedes Alter, jede Rasse und jedes Temperament, des Menschen Gestus und Habitus, seine Sprache und seine Mimik, sein Leben 
und damit sein Sterben -, kurzum: Aufgabe des Arztes ist «die Bestellung des Lebenszustandes» insgesamt, das eben, was ihn zum Zeugen der Szenen des Lebens macht. Leib und Seele stehen dabei in einem Nicht-Ohne-Verhältnis und verhalten sich wie Partner. Es gibt hier kein Hintereinander oder Ineinander, sondern nur das Miteinander der Seele «mit ihrem irdischen Gefährten». Wir finden weder reine Geistesstörungen noch pure Körperkrankheiten: Jedes Organ spricht vielmehr leibhaftig und je spezifisch den Dialekt seiner seelischen Affektion.

Auf dieser theoretischen Basis vermag Nasse dann auch jenes therapeutische großangelegte System aufzubauen, das - weit über die bloße Krankenversorgung hinaus - alle Bereiche eines zu zivilisierenden Lebens umfaßt: die klimatischen Einflüsse von Licht, Luft und Wärme ebenso wie die Wirkungen der Nahrungs- und Genußmittel, die Rolle von Schlafen und Wachen gleicherweise wie das Arbeitsmilieu und den Affekthaushalt, eben jene Natur-Geschichte des gebildeten und zu bildenden Menschen, die das charakterisiert, was Nasse nennt «die eigentliche Anthropologie».

Mit diesem biologischen Lebenskreis aber tritt ein weiteres Phänomen in den Horizont der Wissenschaft: «das Verhältnis des Menschen zum Menschen, und das des Menschen zu allem anderen, womit er das Erdendasein teilt». Hier gilt es zu erforschen, wie Menschen aufeinander wirken, in ihrem Habitus, mit ihrem Sexus, und wiederum je spezifisch nach Alter, Rasse, Temperament, nicht zuletzt jeweils gefärbt durch die Krankheit. Den wirklichen Menschen erfahren wir erst, wenn wir über Natur und Geschichte hinaus den Menschen in seinen sozialen Verbindlichkeiten finden. Das Hauptinstrument dieser Vergesellschaftung aber wird für Nasse nicht die Arbeit, sondern die Sprache, und die Sprache entsteht auch nicht aus den Produktionsverhältnissen, sondern aus dem Geiste der Musik. Sprache aber ist nicht nur ein eminent soziologisches Grundmuster, sondern auch das psychologische Medium, das dem Arzt bei allen Störungen entgegenkommt und somit eine ganze Pathologie und Physiologie zur Voraussetzung hat.

Noch einmal versucht Nasse ihn zu fassen, diesen Logos von der Physis, der über die biologische Struktur des Menschen auch seine sozialen Funktionen umgreift, jene Medizin als Naturkunde, Naturgeschichte und Gesellschaftskunde nämlich, wie sie im Programm einer umfassenden «Anthropologie» zu liegen schien, auch wenn es Nasse nicht gelingen sollte, mit dem Programm und der Paraphrase nun auch eine Architektonik dieses «Kosmos Anthropos» zu liefern; aber das ist ja auch uns nicht gelungen - trotz Freud und Jung, trotz Weizsäcker und so weiter! 
Am Krankenbett freilich verblassen all diese Theorien. Hier läßt Nasse nichts anderes gelten als «die Einschaltung des hundertgliedrigen Lebens in die Krankheit» und daraus folgend: die «Bestellung des Lebenszustandes». Bestellung - schlichter und schöner könnte man die alte «therapeia» nicht übersetzen, die ja stets auch Diakonia war und Epimeleia meint, Dienen und Sorgen im Dienste! Ganz bewußt leitet Nasse in seinem «Handbuch der speziellen Therapie» den Begriff «therapeuo» ab von «ich diene». Heillehre umfaßt ebenso das Verhüten oder Erleichtern wie das Heilen; sie umgreift die privaten Pflegedienste wie auch alle öffentliche Gesundheitspflege.

Selbst der Arzt in persona gilt noch als ein Heilmittel, und zwar als «medicamentum grave», von dem es heißt: «Als Helfer gegen die Krankheit wirkt nur der Arzt», als «iuvamentum» der Arzt als Droge, aber auch der Arzt als Noxe, als ein «nocumentum»! Wer hingegen glaubt, ohne umfassende Ausbildung, lediglich durch gute Absicht oder rednerisches Talent heilen zu können, der ist kein Arzt, sondern - wie Paracelsus das nannte «ein Verwalter des Glücks»; der kann - mit den Worten von Nasse «vielleicht Äußerungen der Kranken, die augenblicklichen Effekt machen, hervorrufen; der Erfolg aber wird zeigen, daß das ein Werk ohne Haltung und Bestand gewesen sei». Es ist auch hier im Grunde wieder das empirische Interesse des Naturforschers, das den Kliniker freihält von den damals überreichlich wuchernden Spekulationen. «Je strenger die Methode», bekennt Nasse, «desto mehr ziemt sie dem Anthropologen.» Daher sein entschiedener Rat: «Jede Vernichtung, jede Daseinsform, die psychologisch betrachtet wird, soll es auch, so weit Tatsachen dazu vorhanden sind, physiologisch, und umgekehrt; und jedes gesondert psychologisch und physiologisch Betrachtete soll auch nachher psychisch-physiologisch zur Erörterung kommen» (1823).

Um es zusammenzufassen: Gegenstand der Anthropologie ist der Mensch, so wie er ist: der Mensch als Natur, als Sozialwesen, als Schicksal, der leibhaftige Mensch mit all seinen Lebenskrisen, die uns dann auch die kritischen Felder des Lebens anbieten, die der Arzt fachkundig zu begleiten hat. Der Arzt begleitet den ganzen Menschen: Zeugung und Geburt, Aufzucht und Wachstum, «die ganze Welt voll mannigfaltiger Zustände und Verhältnisse», jedes Geschlecht, jedes Temperament, Krankheit und Altern, Sterben und Tod. Nicht nur durch die Bekämpfung der Krankheiten, sondern auch durch die Vorsorge für die Gesunden wird der ärztliche Stand wieder jene Stellung in der Gesellschaft erhalten, die er in den alten Heilkulturen so selbstverständlich innehatte. 
Der Arzt wird - so Nasse in seiner programmatischen Schrift über «Die Ärzte im Staate» - einst wiederum werden, was er immer war: «der Berater der Gemeinden, der Gesundheitsfreund, der Lehrer über das, was zur Erhaltung und Bewahrung des Lebens wohl tut, der Fürsorger für die Kinder», und noch einmal: «Den Arzt ruft sein Geschäft zum Zeugen der großen Szenen des Lebens, wo Freude und Trauer, Scheiden und Wiedergewinnen, mächtig die Herzen der Menschen erschüttern» (Von der Stellung der Aerzte im Staate, 1823). Der Arzt ist in der Tat der Zeuge aller großen und kleinen Szenen des Lebens; als Zeuge ist er zugleich aber auch Handelnder und dient damit einer Idee, der Idee nämlich, die «Herrschaft der Gesundheit auf Erden» zu vollenden, um damit die «reine Naturgestalt des Menschen» zu erhalten und wiederherzustellen.

\section{Ausblick}

Wir sollten von dem alten, so liebenswerten Bild vom Arzt als dem Zeugen des Lebens nicht Abschied nehmen, ohne wenigstens einen Blick geworfen zu haben auf die Krisen der Gegenwart und unsere Chancen für die Zukunft. Wir stehen zwischen Gestern und Morgen in einem abenteuerlichen Paradigmawechsel der Medizin und erwarten heute schon, daß die alte, krankheitsorientierte Heiltechnik abgelöst werde von einer neuen, eher krankenorientierten Heilkunde.

Viele Ärzte möchten heute ihre Aufgabe weniger in der Reparatur von Organdefekten sehen als vielmehr in der fachkundigen Begleitung ihrer Kranken. Der Arzt von morgen könnte dabei zum Geburtshelfer eines neuen diätetischen Denkens werden, das seinen eigentlichen Auftrag im Vorfeld der Krankheiten sieht. Eng damit verknüpft wären aber auch die wachsenden Aufgabenfelder für die Patienten, zumal es heute bereits vielfach so ist, daß ein Chronisch-Kranker sein eigener Facharzt wurde. Viele Kranken werden ihren Ärzten und Helfern kundig zur Hand gehen und Rede und Antwort stehen können. Wenn wir heute - vielfach noch unbedacht - vom mündigen Bürger sprechen, so kann damit nur ein Mündigwerden beider Partner gemeint sein, des Leidenden und seiner Helfer, der Kranken wie aller die Kranken begleitenden Berufe im Gesundheitswesen.

Damit hätten wir einige Kriterien und zugleich auch schon Programme angedeutet für eine veränderte Daseinsgestaltung und letztlich einen neuen Lebensstil: 
1. Die neue patientenorientierte Medizin zielt mehr auf Lebensqualität als auf Lebensstandard. Sie beruht auf den anthropologischen Kriterien der Ganzheitlichkeit, der Mitmenschlichkeit und der Verantwortung des Menschen für seine Umwelt.

2. Eine solche am kranken Menschen orientierte Medizin umgreift neben der Umwelt und Arbeitswelt auch die Mitwelt des Menschen im engeren Sinne; sie erstrebt ein humaneres Verhältnis der zwischenmenschlichen Beziehungen.

3. Eine humanökologisch orientierte Heilkunst zielt mehr auf die Resozialisierung des ganzen Menschen als auf die Rehabilitation einzelner Funktionen. Sie bezweckt weniger die «restitutio ad integrum» als eine «restitutio ad integritatem».

4. Die neue krankenorientierte Heilkunde sucht über ihre technischen Möglichkeiten hinaus immer auch die Rechtfertigung aller Eingriffe. Sie basiert weder auf einem zeitlos gültigen Naturrecht noch auf einem gesellschaftlich tolerierten Gewohnheitsrecht, sucht vielmehr das Bezugssystem einer neuen Ethik auf, aus dessen Verantwortlichkeit heraus sie jeweils ihre Entscheidungen für den Alltag zu treffen vermag.

Während uns die Medizin der letzten hundert Jahre als ein vornehmlich auf dem naturwissenschaftlichen Modelldenken beruhendes System der Krankenversorgung und Sozialversicherung imponiert, war die ältere Heilkunde vorrangig an einer Lehre von der Gesundheit orientiert, um erst in zweiter Linie die heiltechnischen Maßnahmen bei der Krankenversorgung zu berücksichtigen. Und auch hier erleben wir in unseren Tagen erst den aufregenden Wandel unserer Gesundheitsvorstellungen: von der Hygiene und Diätetik der älteren Heilkunde über das kurze Intermezzo einer ausschließlichen Krankenversorgung und Sozialversicherung bis hin zu einer von der Medizinischen Pädagogik und Medizinischen Ethik bereicherten Gesundheitsbildung, welche die Medizin wiederum zu einem Modell der Lebensführung und der Weltbewältigung machen könnte.

Dem Panoramawandel der Krankheitslehren und Gesundheitsvorstellungen entspricht der Wechsel der Therapie: von rein empirischen Heilmaßnahmen über die gezielte spezifische Therapie der ätiologisch abgesicherten Infektionskrankheiten bis hin zur lediglich symptomatischen Therapeutik bei den multifaktoriell bedingten und in der Regel chronisch ablaufenden Zivilisationskrankheiten. Wir werden es am Ausgang der 80er Jahre nahezu ausschließlich mit Chronisch-Kranken zu tun haben: mit Langzeitpatienten und - dank den Erfolgen einer immer intensiver werdenden Heiltechnik - 
mit Vielfach-Leidenden (gestützt nicht zuletzt durch die Aufweichung des Krankheitsbegriffs), mit Überlebenspatienten, deren längeres Altern potenziertes Leiden weiterschleppt, mit einer Multimorbidität und Multimedikation und deren Verwicklung in Kettenkrankheiten, mit einem ganzen Heer an Invaliden auf dem Schlachtfelde des zivilisatorischen Stresses, Chronisch-Kranken also, von denen dann - auf die Dauer gesehen - wohl auch jeder sein eigener Arzt werden dürfte.

Mitten in dieser Krise der Medizin stehen wir - so scheint es - vor einem entscheidenden Wandel. In der Vorahnung eines solchen Paradigmawandels sah bereits Friedrich Nietzsche dicht neben die Wissenschaft eine «Gesundheitslehre des Lebens» treten: Die Wissenschaft selber bedarf einer höheren Aufsicht und Überwachung. Wir sind über Kybernetik, Informatik, Semantik längst dabei, aus den Zeichen der alten Wissenschaft eine neue Sprache zu erlernen, die mit Sicherheit nicht mehr die Redeweise der Naturforscher des 19. Jahrhunderts sein wird. Wir haben gerade im Umgang mit Patienten und damit in der Zeugenschaft der kritischen Szenen des Lebens wieder gelernt, daß Krankheiten Schlüssel sind, die uns Tore zum Leben öffnen.

Von solcher Erfahrung berichtet André Gide in seinen Tagebüchern, um daraus zu folgern: «Ich habe unter denen, die sich einer unerschütterlichen Gesundheit erfreuen, noch keinen getroffen, der nicht nach irgendeiner Seite hin ein bißchen beschränkt gewesen wäre, wie solche, die nicht gereist sind.» Wir gleichen - Patienten wie Ärzte - eher jenen, die viel gereist sind, viel auch erfahren mußten, vieles wieder aufzuholen haben, was am Wege liegenblieb, vieles auch noch zu besorgen haben. Für die Ärzte - Theoretiker wie Praktiker - bedeutet dies: daß aus dem Spritzzimmer wieder ein Sprechzimmer werde, daß nicht nur Pillen aufgebracht werden, sondern auch Zuwendung, und daß die Ärzte sich mit der gleichen Sorge und Sorgfalt, mit der sie sich den $30 \%$ der «blutenden» Patienten widmen, sich auch einmal den $70 \%$ der «weinenden» zuwenden sollten.

Wo bleibt in unserer modernen Medizin der Patient, so hat man sich selbstkritisch oft genug gefragt, und die Antwort gegeben: «Für den Arzt ist der Mensch alles.» So hat der Heidelberger Kliniker Ludolf von Krehl es ausgedrückt und diesen seinen Grundgedanken dann auch (1930) weiter ausgeführt: «Wenn der Mensch als Ganzes zum Vorwurf der Forschung dient, da kann man nicht mehr fragen, gehört eben diese Erforschung zu der Naturwissenschaft, zur Biologie, zu den Geisteswissenschaften? Sie braucht sie alle, sie steht zu allen in Beziehung.» 
Ziel einer solchen flächendeckenden ärztlichen Begleitung ist allerdings nicht mehr die «restitutio ad integrum», die den Organdefekt repariert, um den Menschen wieder arbeitsfähig und genußfreudig zu machen. Ziel ist eher die «restitutio ad integritatem», die auf das Ganze eines humanen Lebensentwurfes hinausgeht, die auch die Restfähigkeiten und Leistungsreserven des Behinderten noch so zu mobilisieren vermag, daß eine «optimale Lebensbilanz» bleibt - und das wäre ja schon beinahe ein Begriff für Gesundheit! «Eine Heilung, aus der nur der frühere Zustand wieder hervorgeht und nicht ein starkes Mehr an Lebensgeist, ist eben keine; und andererseits: Erneuerungen eines Wesens, wie wären sie möglich ohne zeitweiliges Erkranken?» (so Hans Carossa im «Arzt Gion»). Der Arzt wäre hier - wie Viktor von Weizsäcker das formuliert hat - weniger ein «Bewirker» als ein «Ermöglicher»: «Er steht nicht über der Entscheidung, sondern mit dem Kranken in der Entscheidung.»

Die Medizin wird unter solchen Kriterien zur Kunst einer äußerst behutsamen Lebensführung, wobei dem Arzt als dem Zeugen des Lebens ebenso fundamentale Aufgaben wie klare Grenzen zufallen. Die Medizin weist nämlich - so der jüdische Arzt Maimonides im arabischen Mittelalter nur hin auf das Nützliche, und sie warnt vor dem Schädlichen; aber sie zwingt weder zu jenem noch straft sie für dieses. Der Arzt ist kein Schulmeister und kein Polizeimann; er sollte sich nicht zum Politiker degradieren noch zum Seelsorger stilisieren lassen. Er kann als der Zeuge der großen und der kleinen Szenen des Lebens nur begleiten und beraten und wird immer nur helfend eingreifen. Die Medizin ist weder Wissenschaft noch Kunst: sie ist ein ebenso persönlicher wie sozialer Auftrag.

\section{Literaturhinweise}

Altmaier, Gottfried, Versuch zu einem systematischen Entwurf der Anthropologie bei Friedrich Nasse, Diss. med. Heidelberg 1977.

Bock, Hans Erhard, Der Arzt und seine Zeit. Therapie im Wandel. Karlsruhe 1978.

Böhme, Wolfgang (Hrsg.), Das Bild des Menschen in der Medizin. Herrenalber Texte 17. Karlsruhe 1979.

Gebsattel, Viktor E. von, Prolegomena einer medizinischen Anthropologie, Berlin/Göttingen/ Heidelberg 1954.

Krehl, Ludolf, Entstehung, Erkennung und Behandlung innerer Krankheiten, Bde.1-3, Berlin 1930-1933.

Nasse, Friedrich, Untersuchungen zur Lebensnaturlehre und Heilkunde, Halle 1818.

- (Hrsg.), Zeitschrift für psychische Aerzte, Bde. 1-5, Leipzig 1818-1822. 
- Die Aufgaben der Anthropologie. Zschr.f. d. Anthrop. 1 (1823) 1-29.

- (Hrsg.): Zeitschrift für die Anthropologie, Bde. 1-4, Leipzig 1823-1826.

- Von der Stellung der Aerzte im Staate, Leipzig 1823.

- Über den Begriff und die Methode der Physiologie, Leipzig 1826.

- Handbuch der speziellen Therapie, 2 Bde., Leipzig 1830/38.

- Anleitung zur Uebung angehender Aerzte in Krankheits-Beobachtungen und Beurtheilung, Bonn 1834.

- Die Isogenesis, ein Naturgesetz, Bonn 1844.

Noorden, Werner von, Christian Friedrich Nasse, ein Vorkämpfer und Wegbereiter des deutschen Arzttums, Berlin 1936.

Paracelsus, i.e. Theophrastus von Hohenheim, Sämtliche Werke. 1.Abt.: Medizinische, naturwissenschaftliche und philosophische Schriften. Hrsg. Karl Sudhoff. Bde.1-14. München/Berlin 1922-1933.

Schipperges, Heinrich, Zum Organismus der Anthropologie bei Christian Friedrich Nasse. Sudhoffs Archiv 59 (1975) 184-201.

- Leitlinien und Grenzen der Psychosomatik bei Friedrich Nasse. Confinia Psychiatrica 2 (1959) 19-37.

- Christian Friedrich Nasse 1778-1851. Medizin in unserer Zeit 1 (1977) 139-142.

- Wege zu neuer Heilkunst. Traditionen, Programme, Perspektiven. Heidelberg 1978.

Steudel, Johannes, Die Stellung der Ärzte im Staate. Die med. Welt 20 (1951) 1231-1233.

Weizsäcker, Viktor von, Der kranke Mensch. Eine Einführung in die medizinische Anthropologie. Stuttgart 1951.

Wunderli, Jörg, und Kurt Weisshaupt (Hrsg.), Medizin im Widerspruch, Olten/Freiburg i. Br. 1977.

Zander, Josef (Hrsg.), Arzt und Patient. Erwartungen und Wirklichkeit. Düsseldorf 1976.

\section{Summary}

The physician is a witness of our life, for he is present in the critical moments of birth, illness, and death. He is the specialist for sufferings and has to avert one's troubles. The Hippocratic physician was a sort of a steersman (kybernetes). He had to regulate the humours of the organism. The Arabian doctor had to accompany his patient and to be his protector against difficulties. Paracelsus says: The human being needs a shepherd. Blessed the patient, whom God gives his physician! - In the 19th century it was Christian Friedrich Nasse (1778-1851), clinician at Bonn, who newly described the duty of the medical man. Nasse demands from him the long observation of a few patients, the careful study of their circumstances, and the consideration of body and soul. The physician himself is a remedy. He must be an "anthropologist", knowing every crisis of the human life. As a friend of health he is present at the great scenes of life. These directives remain worthful for the future.

Prof. Dr. med. Dr. phil. Heinrich Schipperges

Im Neuenheimer Feld 305

D-6900 Heidelberg 